\title{
Modelo de Gestión de un Plan de Desarrollo Productivo Local: El Caso de Nova Friburgo, Brasil
}

\author{
Jesús Domech Moré, Frederico Guilherme Ferreira Lima', Luciene Nascimento de Almeida²
}

\begin{abstract}
The present research looks to analyze the impacts of innovations on the deficiencies listed in the development plan of the Local Productive Arrangement (LPA) of Moda Intima, located in the city of Nova Friburgo, according the perception of the LPA governance. The main ideas of the LPA governance about innovations and management of innovation were initially investigated, firstly in terms of how innovations can improve the performance of the factors which influence the LPA development and secondly in terms of how these factors explain the deficiencies observed in the LPA. Five member of the governance were submitted to two structured questionnaires. Their responses, captured in numeric format, originated a relative impact indicator which was used to asses the impact of innovations on the mentioned deficiencies. The results of the research suggest that the LPA process of innovation is underperformed and therefore has a low impact in alleviating the LPA deficiencies.
\end{abstract}

Key words: innovations; governance; local productive arrangement; impact.

\section{Resumen}

La presente investigación trata del análisis del impacto de la innovación en las deficiencias que aparecen listadas en el Plan de Desarrollo del Sistema Productivo Local (SPL) de Moda Íntima de Nova Friburgo, según la percepción de su Administración. Inicialmente fueron investigadas las principales ideas de la Administración del SPL sobre las innovaciones y nuevas tecnologías. Primeramente en función del desempeño de los factores que influyen en el desarrollo del SPL (fundamentalmente las innovaciones y las nuevas tecnologías) y seguidamente a partir de la influencia de esos factores en las deficiencias presentes en el SPL. Cinco gestores de la Administración fueron sometidos a tres cuestionarios estructurados. Las respuestas, llevadas para un formato numérico, originaron un indicador de impacto relativo el cual fue utilizado para evaluar el impacto que podrían tener las innovaciones en las deficiencias mencionadas. Los resultados de la investigación sugieren que el proceso de innovación posee un débil desempeño en el SPL y ejerce un impacto bajo cuando se trata de atenuar las deficiencias de este.

Palabras claves: innovaciones; administración; sistema productivo local; impacto.

\footnotetext{
${ }^{1}$ Universidade Estácio de Sá, Mestrado em Administração. Avenida Presidente Vargas, 642, 22 andar, sala 2207. Centro. Rio de Janeiro. Brasil. CEP: 2007I-00I. Tel: (552I) 2206-9743 Fax: (552I) 2206-975I. Email: jesus.more@estacio.br, fgl 17@yahoo.com.br

${ }^{2}$ Centro de Tecnologia da Indústria Química e Têxtil (SENAI-CETIQT). Rua Magalhães Castro, I74, Riachuelo, Rio de Janeiro. Brasil. CEP. 2096I. Tel: (552I) 3392-0595/ (5521) 8873- 9224. Email: cienenascimento@ibest.com.br
} 


\section{Introducción}

Hace ya varios años que Schumpeter (1934) publicó las ideas que constituyen, todavía hoy, una referencia de gran relevancia para la comprensión del papel de la innovación tecnológica en el desarrollo económico y que rescata un aspecto fundamental con respecto al agente central de este proceso. Para él, la empresa y la red de relaciones en que esta se encuentra inserida son protagonistas esenciales del proceso de innovación y avance tecnológico. Schumpeter (1939) consideró al emprendedor como aquel que introduce la innovación, crea desequilibrio y provoca el crecimiento económico. Destacó el papel fundamental de la innovación en el acto de emprender y su consecuente impacto en el crecimiento económico.

La innovación tecnológica en Brasil, específicamente en empresas de pequeño y medio porte, todavía es un camino a trillar. Los factores que pueden influir en la innovación de una organización tienen diferentes orígenes y muchos puntos fuertes presentes en una empresa pueden hacer que esta sea más o menos innovativa en sus mercados. En particular, en el Manual de Oslo (2005), se proponen diferentes características y requisitos de las empresas consideradas innovadoras.

Al evaluar los factores internos, externos y estructurales en una empresa brasileña que puedan llevar a una decisión positiva en el sentido de implantar una operación con agregación de valor local, una matriz obviamente debe considerar inicialmente las tendencias en relación a la estabilidad política y económica. Partiéndose de esta premisa atendida, los criterios varían en peso, de empresa a empresa, pero siempre pasan por requisitos como la existencia de un ambiente propicio a la innovación (universidades e institutos de investigaciones), disponibilidad y capacitación tecnológica de recursos humanos, infraestructura moderna, desarrollo y producción local y financiamiento, entre otros. La cuestión de la actitud de los gestores respecto al cambio aparece recogida no Manual de Oslo (2005) como uno de los factores que obstaculizan las actividades de innovación. Este asunto no ha sido explorado en la literatura brasileña.

Buscando entender la importancia de la proximidad geográfica de empresas para la ocurrencia de cooperación y de las fuentes locales de competitividad en relación a la capacidad innovativa y al desarrollo regional, surgieron, por parte de varios autores, diferentes denominaciones, entre ellas: Cluster, Aglomerado, Millieu Innovador, Distritos Industriales, Sistema Productivo e Innovativo Local y Sistema Productivo Local - SPL. En Brasil, el análisis de la dimensión local a partir del concepto de SPL es el que más se destaca.

La Red de Investigación en Sistemas Productivos e Innovativos Locales - RedeSist, del Instituto de Economía de la Universidad Federal de Río de Janeiro, con base en el análisis de un conjunto de 26 empresas productivas en Brasil, realizada a partir de 1998, desarrolló una propuesta de entendimiento de los sistemas productivos locales en los países en desarrollo. El concepto de SPL, adoptado por la RedeSist, está relacionado con el concepto de Sistemas Productivos e Innovativos Locales, definidos como [...] conjunto de agentes económicos, políticos y sociales, localizados en un mismo territorio, desarrollando actividades económicas relacionadas y que presentan vínculos expresivos de producción, interacción, cooperación y aprendizaje (Lastres y Cassiolato, 1999). Los Sistemas Productivos Locales generalmente incluyen empresas - productoras de bienes y servicios finales, suministradoras equipamientos $y$ otros insumos, prestadoras de servicios, comercializadoras, clientes, etc., cooperativas, asociaciones y representaciones - y demás organizaciones dirigidas a la formación y entrenamiento de recursos humanos, información, investigación, desarrollo e ingeniería, promoción y financiamiento. (Lastres y Cassiolato, 2004, p.5).

Partiendo de esta definición, el concepto de sistemas productivos propuesto por la RedeSist está basado en una visión neo-schumpeterina sobre innovación y cambios tecnológicos, que destaca los siguientes factores:

- la innovación y el conocimiento como elementos clave de la dinámica y del crecimiento de países, regiones, sectores, instituciones y organizaciones;

- la innovación como proceso de aprendizaje que depende de interacciones y sufre influencia de ambientes;

- existencia de diferencias entre la capacidad de aprendizaje de los agentes;

- existencia de especificidades en cada país, región, sector, organización, etc; 
- la importancia del conocimiento tácito para el éxito innovativo $y$, al mismo tiempo, la dificultad de transferencia de esos activos.

Una parte sustancial de las investigaciones dedicadas a interpretar las transformaciones socioeconómicas y territoriales asociadas a la nueva fase de crisis capitalista incorpora como referentes centrales a los procesos de innovación y al espacio de interacción conformado por flujos y redes. Se consolida así la idea de que el esfuerzo innovador, que propicia la generación e incorporación de conocimientos para dar respuesta a los retos y problemas a los que las sociedades deben hacer frente, resulta un factor clave que permite no sólo a las empresas sino también a los distintos ámbitos territoriales, insertarse con una mejor posición en un espacio abstracto de redes que interactúa con el concreto de los lugares y que se consolida como lógica espacial dominante.

El ambiente en que los gestores de las empresas están inmersos ya constituye, por si solo, una gran ventaja para las empresas debido a la proximidad física y a las entidades culturales y sociales. Tales ventajas consisten en la presencia de mano de obra especializada, en la facilidad y velocidad de comunicación y en el knowledge spillover - que se refiere al superávit de conocimiento tácito más allá de la empresa extendiéndose al sistema productivo local. (Vasconcelos, Goldszmidt y Ferreira, 2005, p 18). Ese conocimiento no se encuentra en fórmulas y proyectos, es inherente a las habilidades personales y procedimientos operacionales que no se prestan a definiciones explícitas. Atribuyese a ese efecto, entre otros aspectos, la capacidad de rápida difusión de innovaciones a lo largo del SPL, generalmente en función de contactos personales de emprendedores y colaboradores de las empresas.

En un SPL, la organización de empresas y sus instituciones formales (normas y reglas) e informales (normas e costumbres), al suministrar un parámetro para el proceso de toma de decisiones y para las relaciones inter-firmas, desempeña un papel importante en su dinámica. Acciones conjuntas de firmas y agentes locales, emprendidas por procesos de interacción y cooperación, crean nuevas externalidades, generando eficiencia colectiva, economía en costos de transacción y potencialización de la capacidad innovativa.
Para Silva (2002), la manera como el SPL irá cooperar con proyectos específicos está relacionada con el tejido social, donde los hábitos de relacionamiento, la forma como resuelven sus conflictos y los intereses de la comunidad local representan, también, el modo como serán diseñadas las estrategias. En este contexto, las relaciones culturales, políticas, económicas y sociales entre individuos o entre el grupo y el medio de referencia, bien como el sentimiento de pertenencia y el modo específico de actuar en un dado espacio geográfico, asumen un papel muy importante en la creación del conocimiento.

Podemos citar varios factores que incentivan y aceleran el proceso de innovación en las pequeñas y medias empresas brasileñas, sin embargo, un aspecto fundamental de este proceso se refiere al entendimiento de la Alta Administración, su percepción y apoyo. Ella deberá estar altamente sensibilizada para buscar estar alineada con las nuevas tecnologías, crear políticas de expansión, buscar introducirse en nuevos mercados, promover valores innovadores en los colaboradores de la empresa delineando políticas en el sentido de fomentar incentivos a la innovación.

Partiendo del presupuesto que el concepto de innovación y nuevas tecnologías no posee fronteras bien definidas, o sea, es subjetivo y vago, se propone entonces hacer un estudio para conocer el modelo mental de la alta gerencia del SPL de Moda Íntima de Nova Friburgo (municipio de la región serrana de Río de Janeiro). La premisa central de este trabajo se basa en la concepción de que el SPL, por su naturaleza compleja, posee factores determinantes en su formación, diferenciación, interdependencia y flexibilidad. Estos factores poseen determinado desempeño $y$ establecen relaciones con las deficiencias existentes en el SPL que pueden ser conocidas a partir de la mentalidad subjetiva de la alta gerencia.

En Brasil se percibe un interés del actual gobierno en atraer y retener las inversiones así como el diseño de una política industrial en el sentido de fomentar segmentos estratégicos para el país y aumentar las exportaciones pero todavía muchas empresas no se estructuraron internamente para implementar una gestión profesional y continua de tecnología e innovación (DAUSCHA, 2007). Tal vez muchas no están conscientes de la importancia y las ganancias que da la actividad de la innovación tecnológica y esto puede reflejarse en la mentalidad 
subjetiva de la Alta Administración incidiendo sustancialmente en los procesos de toma de decisión, cuando se pretende atacar las deficiencias y contribuir para el desarrollo del SPL.

El objetivo de este trabajo es precisamente hacer un análisis del modelo mental de los gestores del SPL de Moda Íntima de Nova Friburgo en relación al impacto que pudiera tener la innovación en las deficiencias que aparecen listadas en el Plan de Desarrollo del Sistema Productivo Local. Esto por supuesto generará una actitud determinada de la Administración respecto al cambio que se debería dar en relación a la innovación en el SPL.

\section{Caracterización del SPL de Moda Íntima de Nova Friburgo y Región}

El Sistema Productivo Local de Nova Friburgo, especializado en moda íntima, está situado en la región serrana del Estado de Río de Janeiro y dista cerca de 140 $\mathrm{km}$ da capital. La ciudad cuenta con una población de aproximadamente $180 \mathrm{mil}$ habitantes y constituye el principal núcleo del SPL que se expande a través de los municipios de Bom Jardim, Cantagalo Cordeiro, Duas Barras y Macuco.

Desde las primeras décadas del siglo $X X$, la ciudad de Nova Friburgo presenta una tendencia industrial girada para establecimientos industriales que fabrican tejidos. En 1911 fue inaugurada la fábrica de rendas Arp y en los años 20 se instalaron las fábricas textiles Filó, Sinimbu y Ypu. En 1968 la multinacional alemana Triumph Internacional, tradicional fábrica de lingerie, adquirió la empresa Filó, dando inicio a la historia de la ciudad con la industria de confección.

La recesión de los años 80 provocó una drástica desaceleración de las actividades industriales en el país, llevando empresas a demitir un gran número de empleados. La crisis llegó a Nova Friburgo y, en 1982, la Triumph dispensó cerca de 600 funcionarios desactivando más de 150 máquinas de costura. Llevadas por la necesidad, las costureras demitidas comenzaron a producir artesanalmente lingeries para el mercado local, dando origen a un gran número de micro e pequeñas confecciones. Este fenómeno hizo con que la industria de moda íntima de la región pasase de 4 mil trabajadores, en 1980, para más de 22 mil, en 1998.
A pesar de haber sido cerradas aproximadamente 3000 confecciones en los años 90 debido a la carencia de conocimientos administrativos, técnicos y de mercado por parte de estos emprendedores, cerca de 800 confecciones formales permanecieron establecidas. El proceso de organización formal del SPL comenzó en 1997 y en 2002 fue creado el Consejo de Moda que ejerce su Administración.

\section{Factores que Inciden en el Desarrollo de un SPL}

La literatura recoge algunos factores que inciden en el desempeño de un SPL. Estos son: Interrelación y Cooperación; Emprendedorismo; Planificación Estratégica; Innovaciones y Nuevas Tecnologías; Cultura y Valores Regionales; Administración; Créditos de Financiamiento; Costos de Transacción; Capacitación de Mano de Obra y Actuación de los Poderes Públicos.(Valle, 2005)

I.- Interrelación y Cooperación: Amato (2000) consideró que la cooperación inter - empresas lleva a alcanzar varios objetivos, inclusive a combinar competencias, a utilizar know-how de otras empresas, a dividir costos de investigaciones tecnológicas y riesgos al realizarse experiencias en conjunto para explorar las oportunidades.

2.- Emprendedorismo: es un concepto del campo de la administración que resulta en la creación de nuevas oportunidades de empleos en el desarrollo local y tecnológico y en la innovación de productos y servicios. Para que ocurra depende de personas que apliquen sus conocimientos y competencias. Dolabela (2002) no atribuye el éxito del emprendedor únicamente a algunas características de su comportamiento, sino también a un conjunto de atributos. El emprendedor es el individuo que transforma su visión en acción, está atento a las oportunidades y dispuesto a correr riesgos. Es aquél capaz de identificar oportunidades, promover la transformación, el desarrollo y la innovación. Veiga (2005) considera como característica esencial del emprendedor la habilidad para trabajar en equipo y el interés en aprender e innovar. Dejar el esfuerzo emprendedor desarrollarse a partir de una dinámica natural del mercado es ciertamente insuficiente. Programas gubernamentales son instrumentos indispensables para estructurar un ambiente favorable a 
proyectos emprendedores, a la creación de empresas y a la innovación (Maculan, 2003).

3.- Planificación Estratégica: Casarotto y Pires (200I) entienden que la gestión se inicia por la estrategia. Es posible inferir que cualquier organización, conscientemente ó no, adopta una estrategia considerando que la no adopción deliberada puede ser entendida como la propia estrategia. Según el BNDES Banco Nacional de Desarrollo Económico y Social (2005), las referencias bibliográficas que tratan las ventajas competitivas a partir de las estrategias locales enfocan diversos factores que aceleran el desarrollo de SPLs y la transformación de estos en polos de innovación, pero que no siempre están presentes o suficientemente desarrollados.

4.- Innovaciones y Nuevas Tecnologías: La innovación tecnológica y el desarrollo ocurren como resultado de un conjunto complejo de relaciones entre agentes que producen, aplican y distribuyen varios focos de conocimiento, intercambiando entre si informaciones estratégicas que son insumos básicos de sustentación de la red (ROMERO, 2003). La inserción creciente de equipamientos y máquinas con comandos y controles electrónicos en la producción disloca, cada vez más, el eje de la competitividad hacia la gestión de los negocios, lleva a la búsqueda incesante de mejorías de desempeño y remite a la innovación. Sin embargo, bajo ciertas circunstancias, la participación en clusters o SPLs puede retardar a la innovación. El desarrollo de un pensamiento grupal en las empresas pertenecientes al aglomerado puede implicar el refuerzo de comportamientos antiguos y la rigidez, impidiendo la adopción de la mejoría. Ahuja (2000), estudiando como las redes afectan a la innovación, constató que esta situación ocurre en redes con huecos estructurales, o sea, redes con diversos asociados que no interactúan entre si. Este comportamiento perjudica la relación de confianza entre agentes asociados, dificultando el proceso de innovación.

Cambios en el mercado pueden tornar obsoletos activos como las habilidades de los funcionarios, el know-how, las materias-primas $y$ el design. Debido a este comportamiento rígido, los sistemas productivos locales pudieran no soportar la llegada de innovaciones radicales y consecuentemente podrían entrar en decadencia.
5.- Cultura y Valores Regionales: La valorización de la cultura local es un aspecto fundamental en el desarrollo local, una vez que posibilita el mantenimiento $y$ el aprovechamiento de los recursos y de las tradiciones de esta sociedad. Los ritmos de los cambios culturales son lentos. Es necesario muchas veces, que varias generaciones pasen para que se perciban claramente los efectos de la alteración de la cultura y de los hábitos sobre los comportamientos. Según Romero (2003), los componentes culturales tienen gran influencia en el acceso a la información, a la educación, en la formación de sistemas legales y en la organización industrial de una región.

6.- Administración: Para Fischer (1996, p.19), administración "es un concepto plural, que comprende no apenas la sustancia de la gestión sino también la relación entre los agentes envueltos, la construcción de espacios de negociación y los varios papeles desempeñados por los agentes del proceso".

La administración en SPL es definida como [...] diferentes modos de coordinación, intervención y participación en los procesos de decisión locales de los diferentes agentes - Estado, en sus varios niveles, empresas, ciudadanos y trabajadores, organizaciones no gubernamentales, etc. - y a las diversas actividades que envuelven a la organización de los flujos de producción, bien como el proceso de generación, diseminación y uso de conocimientos. (Stainsack, 2006, p. 4),

Según la SEBRAE (2003), la administración es fundamental para la conducción de las actividades en un SPL. Los SPLs deben buscar la organización en relaciones horizontales, en que haya simetría de poder para que, a través de una relación de diálogo, los gestores definan los objetivos comunes, discutan los proyectos y busquen el consenso en la solución de problemas y en el abastecimiento de las necesidades de la colectividad.

7.- Créditos de Financiamiento: Las micro y pequeñas empresas (MPEs) desempeñan un importante papel en la generación de empleos y renta; sin embargo, son preocupantes las altas tasas de mortalidad que presentan. Una de las causas apuntadas por los empresarios es la dificultad de acceso al crédito, en función de la insuficiencia de garantías. Las instituciones financieras también encuentran esa dificultad, pues no consiguen reducir el riesgo de las operaciones debido a la falta de 
garantías y de informaciones confiables sobre el negocio. Ante esta cuestión crítica emerge, en el ámbito del SPL, el concepto de finanzas de proximidad, como aquellas que surgen de los contactos directos con los agentes de producción. Según Abramovay (2003), las organizaciones que emprenden finanzas de proximidad son capaces de convertir redes de relaciones sociales entre individuos, empresas e instituciones en reducción de costos de transacción bancaria. Para Lima, Lima e Matta (2004) el crédito, mientras tanto, debe ser entendido isoladamente como un componente de apoyo al desarrollo del SPL. Antes es necesario que ocurra la estructuración del sistema productivo, que sea definida su administración, estimulado el potencial de cooperación y el fortalecimiento del capital social.

8.- Costos de Transacción: costos debido a la obtención de informaciones, a la negociación, a la toma de decisiones y a las garantías en el cumplimiento de contratos. Para que las decisiones sean, al menos satisfactorias, es necesario que por parte de las empresas se realice una búsqueda de una amplia gama de informaciones y por otra parte que haya un buen conocimiento sobre las implicaciones de las selecciones realizadas. La actuación en SPLs en que ocurre la construcción del conocimiento favorece la reducción de los costos de transacción.

9.- Capacitación de Mano de Obra: Arruda y Andreotti Junior (2008) destacan que la mayoría de las concentraciones productivas tienen origen en la competencia de artesanos inmigrantes, cuyos conocimientos fueron heredados de las generaciones anteriores. La actividad ejercida en un determinado momento crece, el conocimiento es compartido y exfuncionarios acaban montando sus propios emprendimientos. En la mayoría de los casos, no existe la participación actuante de instituciones de enseñanza, investigaciones y desarrollo tecnológico como elemento de apoyo. De esta forma, las empresas deciden por la creación de sus sectores de entrenamiento, con el objetivo de obtener un control más rígido sobre el proceso de aprendizaje, vinculando sus programas de enseñanza a los resultados estratégicos esperados.

Las prácticas y mecanismos de gestión de la información y del conocimiento, juntamente con la capacitación de la mano de obra son esenciales para la creación de un ambiente organizacional propicio a la innovación. El aprendizaje organizacional actúa como elemento de interacción y por tanto está íntimamente asociado al proceso de innovación. Estas prácticas en los SPLs representan ventanas de oportunidades que permiten generar las sinergias necesarias para la aceleración del aprendizaje organizacional, fortaleciendo así, la innovación tecnológica.

10.- Actuación de los Poderes Públicos: Para el Ministerio de Desarrollo, Industria y Comercio Exterior - MDIC, el apoyo a los SPLs está dado por una nueva percepción que se refleja a través de las políticas públicas de desarrollo, en que la dimensión local pasa a ser tratada como un eje orientador de promoción económica y social. La opción estratégica por la actuación en SPLs ocurre, fundamentalmente debido al reconocimiento de que las políticas de fomento a las MPEs son más efectivas cuando son direccionadas a grupos de empresas y no a empresas individualizadas (MDIC, 2004). La alta capacidad de generación de empleos de las MPEs también justifica la decisión por esta política nacional de promoción de SPLs. De esta forma se valoriza la cooperación, el aprendizaje colectivo, el conocimiento tácito y la capacidad innovativa de las empresas e instituciones locales como cuestiones centrales para el aumento de la competitividad sustentable. (MDIC, 2004).

\section{Método}

El método utilizado consta de 7 etapas. Primeramente fueron escogidos 5 gestores del Consejo de la Moda representando a la Administración. Estos ejercerían sus opiniones a través de tres cuestionarios. El primero fue utilizado para identificar el perfil del gestor entrevistado, el segundo fue para conocer el grado de desempeño de factores que inciden en el desarrollo del SPL y el tercero fue referente a la incidencia que estos factores tienen en las deficiencias del SPL según el modelo mental de los gestores. Estas informaciones ayudaron a crear una matriz de impacto que permitió medir la relación entre el estado del factor Innovación y Nuevas Tecnologías y las deficiencias del SPL.

Según informaciones del site Intimafriburgo, el Consejo de la Moda fue creado para discutir cuestiones estratégicas, estando formado por varias entidades asociadas que juntas coordinan las acciones del Polo de Moda Íntima de 
Nova Friburgo y Región. El Consejo representa a la Administración que está compuesta por las siguientes entidades que actúan de forma conjunta, cada una en su ámbito: Empresariales - Federación de Industrias del Estado de Río de Janeiro (FIRJAN); Sindicato de las Industrias del Vestuario (SINDVEST); Capacitación Servicio de Apoyo a las Micro y Pequeñas Empresas (SEBRAE); Servicio Nacional de Aprendizaje Industrial (SENAI) y Universidad del Estado de Río de Janeiro (UERJ); Fomento - SEBRAE y Compañía de Desarrollo Industrial del Estado de Río de Janeiro (CODIN); Crédito - Banco de Brasil; Banco Nacional de Desarrollo Económico y Social (BNDES) y Agencia de Fomento del Estado de Río de Janeiro (Invest-Rio) y Gobierno - Ministerio del Desarrollo de la Industria y del Comercio Exterior (MDIC); Secretaría de Estado del Desarrollo Económico y Turismo (SEDET/RJ) y Alcaldías Municipales de Nova Friburgo, Bom Jardim, Cordeiro, Cantagalo, Duas Barras y Macuco.

Las 7 etapas para el desarrollo del trabajo fueron:

I.- Selección de los gestores de la Administración

La calidad de la evaluación a ser realizada depende directamente del nivel de información y de conocimiento que poseen los entrevistados formados por representantes de SEBRAE, de SENAI, de la Alcaldía de Nova Friburgo (Alc. N. Frib), de FIRJAN y de SINDVEST. Un gran número de investigaciones que utilizan la opinión de pocos agentes, que debido al conocimiento que poseen pudieran ser considerados como expertos, concluyen que esta etapa es fundamental ya que los seleccionados constituyen sensores de información e influyen directamente en la toma de decisión. (OLIVEIRA, JOÃO y MONDLANE, 2008). La selección de los gestores fue hecha por un criterio de conveniencia (disponibilidad de tiempo para las entrevistas, facilidad de acceso al entrevistado). Ellos representan a cuatro de las cinco entidades presentes en el Consejo de Moda (Administración).
2.- Cálculo del peso relativo de cada gestor entrevistado

Como las informaciones de los entrevistados son heterogéneas fue necesario realizar un levantamiento sobre la calificación de los gestores de la Administración del SPL y con esto crear el perfil de ellos, tomándose en consideración los siguientes atributos: tiempo de trabajo con SPL; participación en seminarios, congresos o workshops sobre la temática de SPL; publicación de artículos o libros sobre la temática de SPL; titulación, cargo ○ función gerencial. De esta forma este primer cuestionario tuvo como objetivo atribuir un peso (PAj) a cada gestor (un índice entre 0 e I).

3.- Selección de términos lingüísticos para la evaluación de los factores que inciden en el desempeño de un SPL.

Para evaluar el desempeño de los diez factores relacionadas con el desempeño del referido SPL fueron escogidos los términos: "Óptimo" (valor numérico I); "Bueno" (valor numérico 0,75); "Regular" (valor numérico 0,5); "Mal" (valor numérico 0,25) e "Pésimo" (valor numérico 0 ). Estos términos han sido utilizados por otros autores durante la evaluación de cuestiones subjetivas (MORÉ et al, 2007).

\section{4.- Aplicación de los cuestionarios}

Fueron aplicados dos cuestionarios a los cinco entrevistados. El primer cuestionario tenía como objetivo responder a la siguiente pregunta: ¿Cuál es el grado de desempeño del factor $X$ en el SPL de Moda Íntima de Nova Friburgo e Región? Cada gestor de la Administración expresó su opinión. Al escoger, por ejemplo, el término "Óptimo", el entrevistado de la FIRJAN quiso expresar que el factor Innovaciones y Nuevas Tecnologías (uno de los diez factores que influyen en el desempeño del SPL) tiene un óptimo desempeño y en este caso el valor numérico correspondiente es I. Ya el gestor de la Alcaldía de Nova Friburgo (Alc. N. Frib) difiere y considera que el desempeño de este factor es regular por tal motivo el valor numérico que aparece en la tabla $I$ es 0,5 . De esta forma fueron obtenidos los datos de la Tabla $\mathrm{I}$. 


\begin{tabular}{|c|c|c|c|c|c|}
\hline Factor & SEB & SEN & A. N. F & FIR & SIN \\
\hline Interrelación y Cooperación & 0,75 & 0,75 & 0,75 & I & 0,75 \\
\hline Emprendedorismo & 0,75 & $\mathrm{I}$ & 0,75 & 0,75 & 0,75 \\
\hline Planificación Estratégica & 0,75 & I & 0,75 & I & 0,75 \\
\hline Innovaciones y Nuevas Tecnologías & 0,75 & 0,5 & 0,5 & I & 0,5 \\
\hline Cultura y Valores Regionales & 0,75 & 0,75 & 0,75 & 0,75 & 1 \\
\hline Administración & I & I & $\mathrm{I}$ & I & 0,5 \\
\hline Créditos de Financiamiento & 0,75 & 0,75 & 0,75 & 1 & 0,75 \\
\hline Costos de Transacción & 0,75 & 0,75 & I & 0,5 & 0,75 \\
\hline Capacitación de mano de obra & 0,75 & 0,5 & 0,75 & 0,75 & 0,5 \\
\hline Actuación de los Poderes Públicos & 0,75 & I & 0,75 & 0,5 & 0,25 \\
\hline
\end{tabular}

SEB.= SEBRAE; SEN.= SENAI; A.N.F.= Alcaldía de Nova Friburgo; FIR.= FIRJAN; SIN.= SINDVEST

Tabla I- Evaluación de los Factores de Desarrollo del SPL por los gestores de la Administración del SPL.

5.- Agregación de las opiniones de los gestores entrevistados

Las opiniones fueron substituidas por un valor numérico (VNj) según la etapa 3. Considerando el peso de cada uno de los gestores $(P A j)$ fue calculada la media ponderada a partir de la agregación de los valores. Para ello fue utilizada la fórmula de la media ponderada que aparece a continuación.

$$
\begin{gathered}
I_{\text {eval }}=\sum_{j=1}^{5} P A_{j} * V N_{j} \\
I_{\text {eval }}=P A_{1} * V N_{1}+P A_{2} * V N_{2}+P A_{3} * V N_{3}+P A_{4} * V N_{4}+P A_{5} * V N_{5}
\end{gathered}
$$

Los resultados aparecen representados en la Tabla 2.

\begin{tabular}{lll}
\hline Factor & Índice de Evaluación & Evaluación \\
\hline Interrelación y Cooperación & 0,75 & Punto fuerte \\
Emprendedorismo & 0,75 & Punto fuerte \\
Planificación Estratégica & 0,83 & Punto fuerte \\
Innovaciones y Nuevas Tecnologías & 0,62 & Punto débil \\
Cultura y Valores Regionales & 0,79 & Punto fuerte \\
Administración & 0,87 & Punto fuerte \\
Créditos de Financiamiento & 0,75 & Punto fuerte \\
Costos de Transacción & 0,73 & Punto débil \\
Capacitación de mano de obra & 0,64 & Punto débil \\
Actuación de los Poderes Públicos & 0,61 & Punto débil \\
\hline
\end{tabular}

Tabla 2. Evaluación de los Factores de Desarrollo presentes en el SPL considerando el peso de los gestores de la Administración. 
6.- Definición de los Puntos Débiles y Fuertes

Los resultados de índices de evaluación que aparecen en la Tabla 2 fueron utilizados en la definición de los puntos fuertes y débiles del SPL. Como referencia se consideró el criterio subjetivo de que todo factor evaluado con un índice resultante menor que 0,75 fuese clasificado como un punto débil $\left(I_{\text {eval }}<0,75\right)$. A partir de esta clasificación los puntos débiles en el SPL fueron: Innovación y Nuevas Tecnologías, Costos de Transacción, Capacitación de la Mano de Obra y Actuación de los Poderes Públicos. Todo factor evaluado con un índice resultante mayor ó igual que 0,75 fue clasificado como un punto fuerte $\left(I_{\text {eval }}<0,75\right)$. Estos fueron: la interrelación y cooperación, el emprendedorismo, la planificación estratégica, la cultura y los valores regionales, la Administración y los créditos de financiamiento.

\begin{tabular}{|c|c|c|c|c|c|c|c|c|c|}
\hline Factor & $\mathbf{A}$ & B & C & D & $\mathbf{E}$ & $\mathbf{F}$ & G & $\mathbf{H}$ & $\begin{array}{l}\text { Suma } \\
\text { (Y) }\end{array}$ \\
\hline I. Interrelación & 0,715 & 0,65 & 0,82 & $0,7 \mid 25$ & 0,955 & 0,5775 & 0,4725 & 0,85 & 5,75 \\
\hline \multicolumn{10}{|l|}{ Cooperación } \\
\hline 2. Emprendedorismo & 0,87 & 0,665 & 0,80 & 0,415 & 0,83 & 0,62 & 0,5 & 0,825 & 5,52 \\
\hline \multicolumn{10}{|l|}{ 3. Planificación } \\
\hline Estratégica & 0,545 & 0,64 & 0,535 & 0,485 & 0,3675 & 0,545 & 0,46 & 0,825 & 4,40 \\
\hline \multicolumn{10}{|l|}{ 4. Innovaciones y Nuevas } \\
\hline Tecnologías & 0,45 & 0,765 & 0,255 & 0,315 & 0,25 & 0,44 & 0,6225 & 0,45 & 3,54 \\
\hline \multicolumn{10}{|l|}{ 5. Cultura y Valores } \\
\hline Regionales & 0,71 & 0,505 & 0,165 & 0,125 & 0,327 & 0,125 & 0,305 & 0,6625 & 2,92 \\
\hline 6. Administración & 0,595 & 0,635 & 0,4225 & 0,915 & 0,7925 & 0,915 & 0,25 & 0,4725 & 4,99 \\
\hline 7. Créditos & & & & & & & & & \\
\hline Financiamiento & 0,665 & 0,665 & 0,7025 & 0,83 & 0,6625 & 0,5 & 0,2875 & 0,21 & 4,52 \\
\hline \multicolumn{10}{|l|}{ 8. Costos de Transacción } \\
\hline & 0,645 & 0,635 & 0,815 & 0,255 & 0,255 & 0,435 & 0,125 & 0,545 & 3,71 \\
\hline \multicolumn{10}{|l|}{ 9. Capacitación de mano } \\
\hline de obra & 0,15 & 0,79 & 0,125 & 0,085 & 0,2475 & 0,835 & 0,855 & 0,915 & 4,00 \\
\hline \multicolumn{10}{|l|}{ 10. Actuación de los } \\
\hline Poderes Públicos & 0,555 & 0,465 & 0,325 & 0,795 & 0,425 & 0,5725 & 0,09 & 0,215 & 3,44 \\
\hline Suma (X) & 5,9 & 6,41 & 4,96 & 4,93 & $5, I I$ & 5,56 & 3,96 & 5,97 & \\
\hline
\end{tabular}

Tabla 3- Representación de la Matriz de Impacto del Factor de Desarrollo 'versus' las Deficiencias del SPL.

7.- Creación de la Matriz de Impacto del Factor de Desarrollo del SPL 'versus' Deficiencias presentes en el SPL.

Un tercer cuestionario fue aplicado a los cinco gestores de la Administración con la intención de extraer el conocimiento en relación al grado de influencia que cada factor ejerce sobre cada una de las deficiencias que aparecen listadas en el Plan de Desarrollo del Sistema Productivo Local (Plano De Desenvolvimento do Apl de Confecção de Nova Friburgo e Região, 2008). Los gestores respondieron a la pregunta: ¿Cuál es el grado de influencia del factor $X$ en la deficiencia $Y$ ? Donde $X$ representa cada uno de los diez factores de desarrollo del SPL y $Y$ las siguientes deficiencias: A - Insatisfactoria experiencia de los empresarios en relación a la identificación de los mercados potenciales, en especial el mercado internacional; B - Dificultades con la innovación y el design; C - Dependencia en relación al suministro de tejidos y otros insumos; D - Recursos propios para el capital de giro y dificultad de acceso al crédito; E Instrumentos de marketing y promoción comercial prácticamente inexistente; F - Costos de producción 
elevados; G - Calidad inferior comparada a las grandes marcas; $\mathrm{H}$ - Falta de patrones en los modelos.

Para evaluar las influencias, los gestores escogieron una de las siguientes alternativas de respuestas: "Total influencia" (valor numérico I); "Posee influencia" (valor numérico 0,75); "Moderada influencia" (valor numérico 0,5 ); "Poca influencia" (valor numérico 0,25); "Ninguna influencia" (valor numérico 0). (Valle, 2007). La tabla 3 muestra el grado con que las deficiencias presentes en el Sistema Productivo Local de Moda Íntima de Nova Friburgo y Región son influenciadas por los factores de desarrollo del SPL, considerando las opiniones de los gestores de la Administración y el peso de cada uno de estos. La Suma (X) representa la sumatoria de las informaciones referentes a cuánto puede estar siendo influenciada determinada deficiencia presente en el SPL por los factores que inciden en su desarrollo. La Suma (Y) se refiere a la sumatoria de las informaciones relacionadas al peso que tiene cada factor en las deficiencias del SPL como un todo.

\section{Resultados y Discusión}

Se observa que el valor de la Suma $(X)=6,4$ I significa que la deficiencia "Dificultades con la innovación y el design" es la que más está siendo influenciada por todos los factores de desarrollo del SPL. El factor que más influye en esta deficiencia es la capacitación de la mano de obra, con un índice de 0,79. Significa esto que la capacitación de la mano de obra que fue considerada por los gestores de la Administración como un punto débil del SPL (ver tabla 2) ejerce influencia en esta deficiencia.

Si consideramos que:

$$
\sum_{d=1}^{8} \operatorname{Suma}(X)_{d}=5,9+6,41+4,96+4,93+5,11+5,56+3,96+5,97=42,8
$$

Donde d son las deficiencias que varían de 1 a 8 , tendremos un valor que representa cuánto el total de deficiencias está siendo influenciado por los diez factores de desarrollo del SPL. Al dividir 6,4I por el valor total 42,8 obtenemos 0,1497. Este valor significa que los diez factores de desarrollo del SPL estudiados aquí ejercen un impacto relativo de aproximadamente 15\% del total en la deficiencia: "Dificultades con la innovación y el design".

Al considerar que:

$$
\sum_{f=1}^{10} \operatorname{Suma}(Y)_{f}=5,75+5,52+4,40+3,54+2,92+4,99+4,52+3,71+4,00+3,44=42,79
$$

Tendremos un valor que representa el total de las influencias de los factores de desarrollo del SPL $(f=1$ hasta 10 ) en todas las deficiencias. Al dividir 3,54 por el valor total 42,79 obtenemos 0,0829 . Este valor significa que el factor Innovaciones y Nuevas Tecnologías ejerce un impacto relativo de $8,29 \%$ del total en todas las deficiencias. A pesar de ser evaluado el factor de desarrollo del SPL Innovaciones y Nuevas Tecnologías por los gestores de la Administración como un factor débil, este impacto $(8,29$ de 100$)$ es considerado como bajo. 


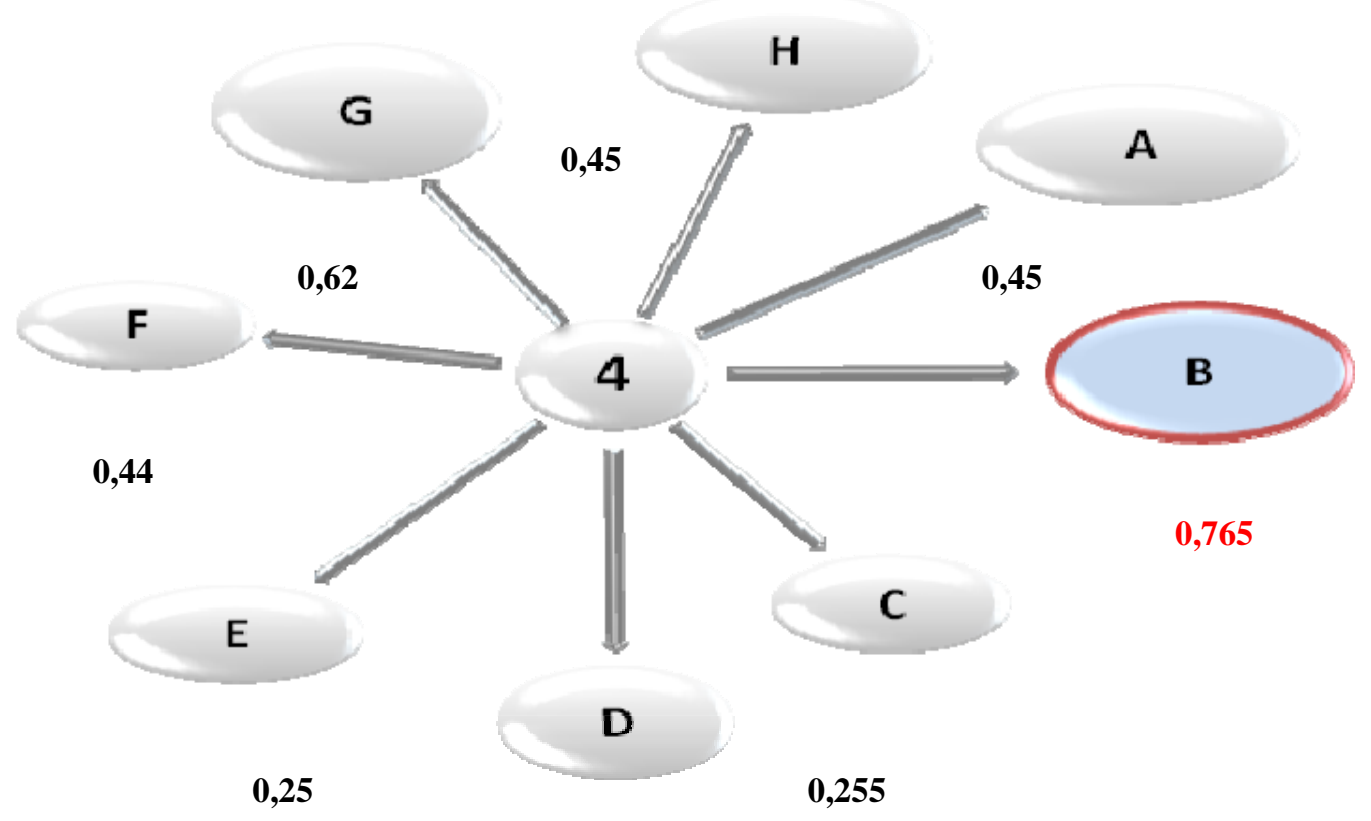

0,315

Figura I. Grados de influencia que ejerce el factor Innovación y Nuevas Tecnologías en las deficiencias del SPL de Moda Íntima de Nova Friburgo y Región.

La figura I representa el grado de influencia que el factor 4. - Innovación y Nuevas Tecnologías - ejerce en las deficiencias $A, B, C, D, E, F, G$ y $H$ del $S P L$ en estudio. Es evidente que el factor Innovación y Nuevas Tecnologías, con índice 0,765 ejerce influencia en la deficiencia "Dificultades con la Innovación y el design".

De manera particular llama la atención el modelo mental de los gestores cuando al agregar sus opiniones constatamos que el factor Innovación y Nuevas Tecnologías ejerce poca influencia en las deficiencias "Dependencia en relación al suministro de tejidos y otros insumos", "Recursos propios para el capital de giro y dificultad de acceso al crédito" e "Instrumentos de marketing y promoción comercial prácticamente inexistente".
Una lectura de este modelo mental nos presenta que el factor Innovación y Nuevas Tecnologías, ejerce influencia casi moderada en las deficiencias "Insatisfactoria experiencia de los empresarios en relación a la identificación de los mercados potenciales, en especial el mercado internacional", "Falta de patrones en los modelos" y "Costos de producción elevados". Finalmente este factor ejerce una influencia un poco mayor que moderada en la deficiencia "Calidad inferior comparada a las grandes marcas".

A pesar de ser evaluado el factor Innovaciones y Nuevas Tecnologías por los gestores de la Administración como un factor débil, este impacto $(8,29$ de 100) está entre los tres más bajos (ver figura 2). 

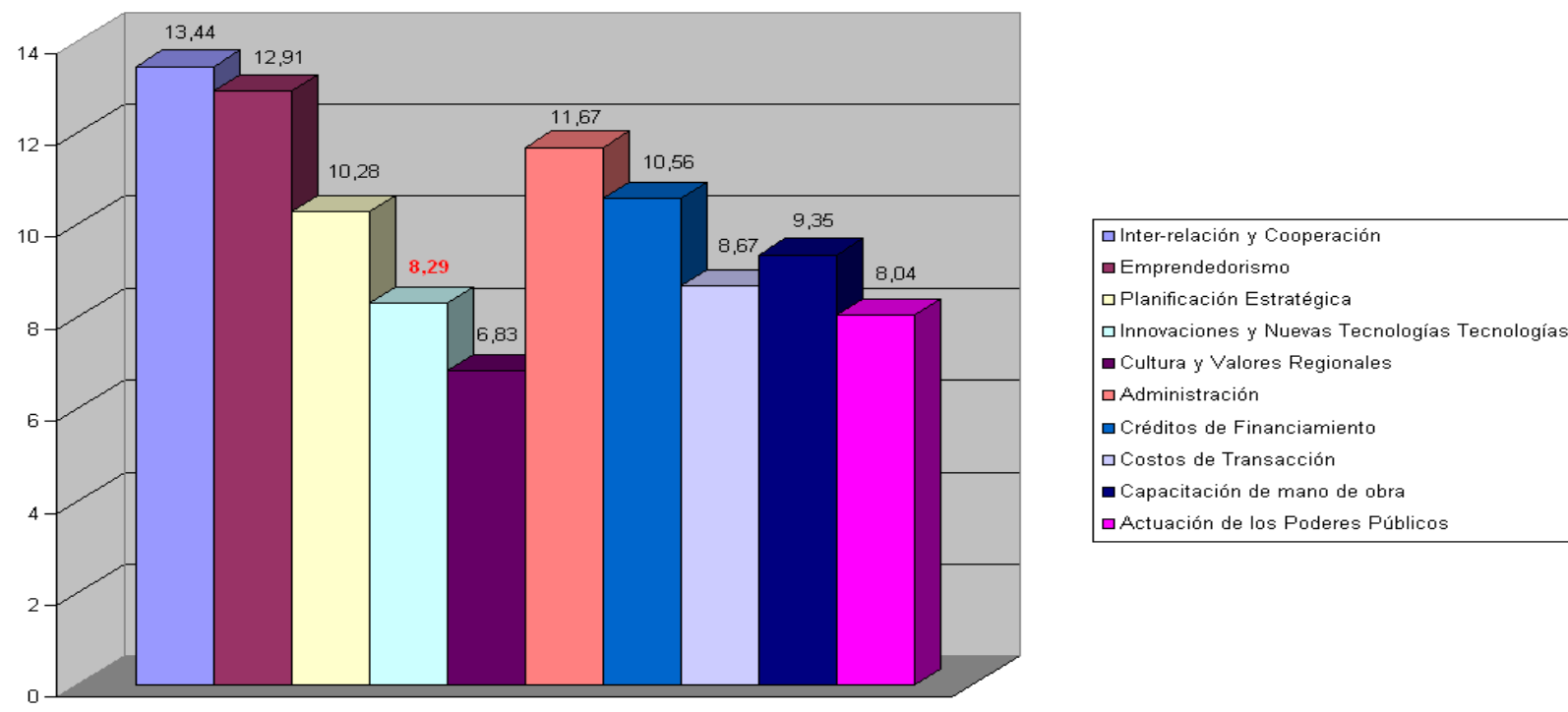

Figura 2. Distribución de los Impactos Relativos de los Factores de Desarrollo del SPL en las Deficiencias de este.

Esto significa que en un proceso de toma de decisión dirigido a atacar las deficiencias del SPL, de forma intuitiva, la Administración no debería aplicar recursos en el factor Innovación y Nuevas Tecnologías. Dicho de otra forma, las innovaciones y las nuevas tecnologías no son consideradas como factores capaces de atenuar las deficiencias presentes en el SPL.

\section{Conclusiones}

Este trabajo realiza un estudio sobre el modelo mental de la Administración del Sistema Productivo Local de Moda Íntima de Nova Friburgo y Región ubicado en el Estado de Río de Janeiro, Brasil. Primeramente se determinaron las debilidades y fortalezas del SPL. Después se consideró, a través de una matriz de impacto, cuánto influye el estado de cada factor de desarrollo del SPL en las deficiencias listadas en el Plan de Desarrollo del SPL.

Este estudio, inédito en la literatura brasileña, permite afirmar que según el modelo mental de los gestores de la Administración del SPL el factor Innovación y Nuevas Tecnologías que posee un débil desempeño dentro del SPL (Índice de evaluación de 0,62), ejerce un impacto bajo en las deficiencias que aparecen relacionadas en el Plano de Desarrollo del SPL (valor de 8,3\%).
Con este resultado es posible concluir que independientemente de que se acepte por parte de los gestores que la innovación es central para el crecimiento de la productividad, todavía el entendimiento sobre el impacto económico en las micro y pequeñas empresas es deficiente. Una comprensión conceptual adecuada de la estructura y las características del proceso por parte de los gestores es fundamental para la formulación y desarrollo de políticas de suporte. De esta forma se corrobora que: "El conocimiento de los gestores es cada vez más percibido como un conductor central de la innovación" (Manual de Oslo, 2005). La percepción que tengan los gestores influirá en la aplicación o no de recursos dirigidos a inversiones o no en procesos de innovaciones y/o asimilación de nuevas tecnologías.

El trabajo constituye un intento de modelar el pensamiento de los gestores en relación a factores del desempeño del SPL, las deficiencias presentes en el mismo y la relación con el factor de innovación. Llama la atención a que esta cuestión subjetiva deba ser considerada como una posible barrera al desarrollo de la innovación en un SPL. Se recomienda, para trabajos futuros, el uso de modelos borrosos en la medición de la subjetividad presente en las opiniones. 


\section{Referencias}

ABRAMOVAY, R. (2003). Finanças de proximidade e desenvolvimento territorial no semi-árido brasileiro. http://www.econ.fea.usp.br/abramovay/artigos cientificos.h tm [Accesado 10/10/2009]

AHUJA, G. (2000). Collaboration Networks, Strutural Holes and Innovation: A Longitudinal Study. Administrative Science Quarterly, 45, 425-455.

AMATO, J. N. (2000). Redes de cooperação produtiva e clusters regionais: oportunidades para as pequenas e médias empresas. Atlas, São Paulo.

ARRUDA, M., Andreotti Junior, W (2006). Um modelo de Universidade Corporativa para Micro e Pequenas Empresas. www.educor.desenvolvimento.gov.br [Accesado 03/10/2009]

BNDES (2005). Arranjos Produtivos Locais, Política e Desenvolvimento. Rio de Janeiro.

CASAROTTO, F. N., Pires, L. H. (200I). Redes de pequenas e médias empresas e desenvolvimento local: Estratégias para a conquista da competitividade global com base na experiência italiana. Atlas, São Paulo.

CONSELHO DE MODA DO APL DE CONFECÇÃO DE NOVA FRIBURGO E REGIÃO (2005). Plano de Desenvolvimento do APL de Confecção de Nova Friburgo e Região. http://www.mdic.gov.br/arquivos/dwnl 1248288038.pdf [Accesado 10/05/2008]

DAUSCHA, R.M.(2005) Innovação Tecnológica no Brasil: um caminho a trilhar. http://www.portalbmk.org.br/publicacoes/arquivos/I I 2290 5848.doc [Accesado 13/09/2009]

DOLABELA, F. (2002). Empreendedorismo. Uma forma de ser. Cultura Editores, São Paulo.

FISCHER, T. (1996). Poder Local: Um tema em Análise. In: Fischer, T. (org.). Poder Local: Governo e Cidadania. FGV, Rio de Janeiro. Pp. 10-19.

INTIMAFRIBURGO (2009). Conselho da Moda - O que é?. www.intimafriburgo.com.br [Accesado 07/06/2009]
LASTRES, H. M. M., Cassiolato, J. E. (1999). Redesist Rede de Pesquisa em Sistemas e Arranjos Produtivos e Inovativos Locais.. http://www.redesist.ie.ufri.br [Accesado I3/09/2009]

LASTRES, H. M. M., Cassiolato, J. E. (2004). Políticas para Promoção de Arranjos Produtivos e Inovativos Locais de Micro e Pequenas Empresas: vantagens e restrições do conceito e equívocos usuais. Rio de Janeiro: UFRJ. http://redesist.ie.ufrj.br/dados/nt.php?projeto=ar I

[Accesado 15/09/2009]

LIMA, A. M., Lima, C. L. C., Matta J. P. (2004). Finanças de Proximidade: Um Modelo de Financiamento para Arranjos Produtivos Locais. http://www.desenbahia.ba.gov.br/ recursos/news/video/\%7B06D3ACB9-706I-4A45-862DD79A3CD546F9\%7D Finan\%C3\%A7as\%20de\%20proximid ade\%20-\%20um\%20modelo\%20de\%20financiamento \%20para\%20APLs.pdf [Accesado 09/10/2009]

MACULAN, A. (2003). Ambiente empreendedor e aprendizado das pequenas empresas de base tecnológica. In: Pequena empresa: Cooperação e Desenvolvimento. Rio de Janeiro: Relume Dumará, UFRJ.

MINISTÉRIO DO DESENVOLVIMENTO, INDÚSTRIA E COMÉRCIO EXTERIOR - MDIC (2004) - Termo de Referência para Política Nacional de Apoio ao Desenvolvimento de Arranjos Produtivos Locais. http://www.desenvolvimento.gov.br/portalmdic/arquivos/d wnl 1234/81254.pdf [Accesado 10/10/2009]

MORÉ, J. D., Valle M. R., Villela., L. E (2007). Fuzzy Model of influences among the endogenous and exogenous factors of one local productive arrangement. In: SIGEF 2007, Proceedings of the XIV Congress of International Association for Fuzzy-Set Management and Economy, I-3 November 2007, Poiana Brasov, Romania.

MORÉ, J. D., Lima, F.G.F., Purificação A.B.A. (2008). O modelo mental da governança do APL de Moda Íntima de Nova Friburgo sobre Cultura, Empreendedorismo e Inovação. InXXV Simpósio de Gestão da Inovação Tecnológica. "Inovação, Cultura e Empreendedorismo". 22 a 24 de outubro de 2008. Hotel Grand Bittar. Brasília, DF.

OCDE (2005). Manual de Oslo - Diretrizes para coleta e interpretação de dados sobre inovação. Diretrizes para 
coleta e interpretação de dados sobre inovação. $3^{\circ}$ ed. FINEP, Sao Paulo.

OLIVEIRA P.H., João H.F., Mondlane N.S. A (2008). Contexto competitivo, monitoramento ambiental e tomada de decisão estratégica: o caso dos micro e pequenos varejos da região do Barro Preto em Belo Horizonte. Revista Ciência da Informação. 37(2), mar-aug.

ROMERO, E. I. C. (2003). Desenvolvimento de clusters a visão do conhecimento proposta do modelo PRUGI de análise de oportunidades para o norte do estado do Rio de Janeiro. Tese de Doutorado, COPPE/UFRJ.

SANTOS, G. A. G., Diniz, E. J., Barbosa, E.K. (2005). Arranjos Produtivos Locais, Política e Desenvolvimento. BNDES, Rio de Janeiro.

SCHUMPETER, J. A. (1934). The theory of economic development. Harvard University, Massachusetts.

SCHUMPETER, J. A. (1939). Business cycles. McGraw Hill Books, New York.

SEBRAE - Serviço de Apoio às Micro e Pequenas Empresas. (2003). Termo de Referência para Atuação do Sistema SEBRAE em Arranjos Produtivos Locais. SEBRAE, 17, Brasília.

STAINSACK, C. (2006). Governança em Arranjos Produtivos Locais: Experiências do Paraná. Curitiba. http://www.ielpr.org.br/apl/uploadAddress/GovernanaAPLs -Set06\%5B47828\%5D.pdf. [Accesado 09/10/2009]

SILVA, G. (2002). Sobre a "Tropicalização" da experiência dos distritos industriais italianos. In: Cocco, G., Urani, A., Galvão, A. P. (Org.). Empresários e empregos nos novos territórios produtivos: o caso da terceira Itália. DP\&A, Rio de Janeiro. Pp. 253-269.

VALLE, M. R. (2005). A utilização da lógica fuzzy para análise de fatores endógenos e exógenos em um arranjo produtivo local: $O$ caso da Moda Praia de Cabo Frio. Dissertação de Mestrado. UNESA, Rio de Janeiro.
VASCONCELOS, F. C., Goldszmidt, R. G. B., Ferreira F.C. M (2005). Arranjos Produtivos. Revista GV Executivo, 4(3). ago-out. 17-21.

VEIGA, D. R. (2005) Características Essenciais à Formação de Redes de Empreendedores no APL de Confecções de Roupa Íntimas de Nova-Friburgo - RJ: uma análise comparativa entre os pressupostos teóricos e a realidade local. Dissertação de Mestrado - UNESA, R.J 